\title{
Clinical Evaluation of Bioabsorbable Mesh for Secondary Bone Grafts in
} the Alveolar Cleft

\section{Tatsuo Shirota ${ }^{1 *}$, Hiroshi Ogura ${ }^{2}$, Maiko Suzuki ${ }^{1}$, Ayako Akizuki ${ }^{1}$, Takaaki Kamatani ${ }^{1}$, Seiji Kondo ${ }^{1}$ and Tetsutaro Yamaguchi ${ }^{3}$}

${ }^{1}$ Departments of Oral and Maxillofacial Surgery, School of Dentistry, Showa University, Tokyo, Japan

${ }^{2}$ Department of Information Science, Faculty of Arts and Sciences at Fujiyoshida, Showa University, Tokyo, Japan

${ }^{3}$ Department of Orthodontics, School of Dentistry, Showa University, Tokyo, Japan

\begin{abstract}
Purpose: This study investigates whether a poly-L-lactic acid (PLLA)-polyglycolic acid (PGA) mesh-type bone joining material (PLLA-PGA mesh), a bioabsorbable osteosynthetic material, can replace titanium mesh for secondary bone grafts in the alveolar cleft.

Methods: The subjects were 6 patients who underwent secondary bone grafting in the alveolar cleft using the PLLA-PGA mesh and 6 patients treated with titanium mesh. Intraoral X-ray images and dental small-field CT (3DX) were taken over a period of 6 months postoperatively. Analysis of Covariance (ANCOVA) was performed for two objective variables obtained from CT images namely the vertical height of the bony bridge and the labial-lingual thickness. A generalized linear model was also used to analyze a discrete objective variable, i.e., the score for the bony bridge height obtained from the occlusal X-ray image. In these analyses, the five explanatory variables used were mesh type, sex, age, grafted bone mass, and width of the alveolar cleft measured on the CT image.
\end{abstract}

Results: No significant factors were observed among the 5 explanatory variables with regard to influence on bony bridge thickness. Mesh type was the only significant factor influencing bony bridge height, and the height was greater when using PLLA-PGA mesh than when using titanium mesh. However, there were no significant factors influencing alveolar crest height on occlusal X-ray images.

Conclusions: There were no marked differences in bony bridge morphology in the alveolar cleft area between the PLLA-PGA and titanium mesh groups.

Keywords: Secondary bone graft; Alveolar cleft; Bioabsorbable mesh; CT image

\section{Introduction}

Secondary bone grafting in the alveolar cleft is performed to improve alveolar shape, guide permanent tooth eruption or move permanent teeth in the alveolar cleft area, and improve a concaved base in the nasal wing [1]. Such bone grafting is typically conducted 6-9 years before canine tooth eruption [2]. However, in some cases, patients obtain their permanent dentition without receiving bone grafts at the appropriate age. To perform secondary bone grafting in the alveolar cleft area with permanent dentition, alveolar shape reconstruction with sufficient height and thickness is required for dental implant treatment. Since many such cases include extensive alveolar cleft or hard scar tissue around the alveolar cleft with high-tension soft tissue, there is a risk of bone resorption from the labial side after bone grafting. Titanium mesh is generally used in such bone grafting cases due to the straightforward formation of a three-dimensional (3D) alveolar shape [3-5]. However, further surgery is required to remove the mesh.

In recent years, bioabsorbable bone-joining materials made from poly-l-lactic acid (PLLA) polymer [6] and PLLA-PGA copolymer consisting of PLLA and polyglycolic acid (PGA) have been developed $[7,8]$. These materials do not need to be removed after healing, and they are therefore widely used in the orofacial area, particularly in cases of fracture and in orthognathic surgery. PLLA-PGA mesh-type bone joining materials can be formed in water at $60^{\circ} \mathrm{C}$, and they are hydrolyzed and absorbed in the body within one year. This material has been widely used for fractures of thin facial bones such as the orbital floor [8]. Although we used titanium mesh conventionally, we noted these characteristics of PLLA-PGA mesh, and since March 2009, we have been using it in patients who require alveolar ridge extension by secondary bone grafting in the alveolar cleft area, but who do not wish to undergo removal surgery.
In the present study, the shape of the bone bridge formed in the alveolar cleft area was compared between PLLA-PGA mesh type and titanium bone-joining materials when these materials were used for bone grafting.

\section{Patients and Methods}

The present retrospective study was performed with the approval of the Ethics Committee of the School of Dentistry, Showa University, Tokyo, Japan.

\section{Patients}

PLLA-PGA and titanium meshes were used for patients with unilateral cleft lip and palate who underwent secondary bone grafting between March 1, 2009 and August 31, 2010 to form the alveolar ridge during bone grafting. The bone bridge shape in the cleft area was assessed on bisector occlusal X-ray images and CT at 6 months postoperatively.

The subjects were 12 patients ( 6 females and 6 males). Age at the

*Corresponding author: Tatsuo Shirota, DDS, Ph.D, Department of Oral and Maxillofacial Surgery, School of Dentistry, Showa University, 2-1-1, Kita-senzoku, Ohta-ku, 145-8515 Tokyo, Japan, Tel: +81-3-3787-1151; Fax: +81-3-5498-1543; E-mail: tshirota@dent.showa-u.ac.jp

Received November 11, 2015; Accepted December 29, 2015; Published January 06, 2016

Citation: Shirota T, Ogura H, Suzuki M, Akizuki A, Kamatani T, et al. (2016) Clinica Evaluation of Bioabsorbable Mesh for Secondary Bone Grafts in the Alveolar Cleft. Dentistry 6: 353. doi:10.4172/2161-1122.1000353

Copyright: $\odot 2016$ Shirota T, et al. This is an open-access article distributed under the terms of the Creative Commons Attribution License, which permits unrestricted use, distribution, and reproduction in any medium, provided the original author and source are credited. 
time of bone grafting in the alveolar cleft area ranged from 14 to 54 years (mean, 25.3 years).

\section{Mesh type and application}

PLLA-PGA mesh (LactoSorb Mesh; Lorenz Surgical Inc., Jacksonville, FL), a PLLA-PGA copolymer absorbable mesh, was used for bone grafting. This mesh is a copolymer composed of $82 \%$ PLLA and 18\% PGA. The thickness of the mesh was $0.5 \mathrm{~mm}$, and 100 holes of $1.5 \mathrm{~mm}$ in diameter were formed on a $50 \times 50 \mathrm{~mm}$ PLLA/PGA membrane. The PLLA-PGA mesh was softened in $60^{\circ} \mathrm{C}$ hot water and pressed onto a template to form the desired shape. A Hex head screw (LactoSorb Screw; Lorenz Surgical Inc.) was used to fix the mesh in the cleft jaw area. The titanium mesh was a micromesh plate with a $0.2 \mathrm{~mm}$ thickness (KLS Martin GmbH \& Co., Umkirch, Germany), and the size and shape were directly adjusted in the cleft jaw area. The mesh was fixed using a micro screw (Centre Drive ${ }^{\star}$; KLS Martin GmbH \& Co.).

The need for mesh during bone grafting was thoroughly explained to the patients, and their consent was obtained. The type of mesh was selected randomly.

\section{Bone grafting in the alveolar cleft}

Bone grafting in the alveolar cleft was performed by 2 operators, and surgery in the alveolar cleft area and graft bone collection from the iliac bone were performed simultaneously. Surgery in the alveolar cleft area was performed by the same operator.

Subperiosteal injection was performed in the surgical area using $2 \%$ lidocaine including 200,000-fold dilution epilenamine. Following a mucoperiosteal incision along the cleft, the gingival mucoperiosteal flaps were reflected onto the nasal side, and the nostril floor was restored. The mesh was adjusted to the alveolar cleft to create sufficient space for bone grafting.

Particulate cancellous bone marrow (PCBM) was collected from the outer side of the iliac crest front margin. A $10 \mathrm{~mm}$ incision was made parallel to the wrinkle to create space for a bone curette, and PCBM was harvested. PCBM was crushed, and its weight was measured with an electric scale. The osteoplastic flap was replaced and secured with a 3-0 resorbable suture, and the wound was closed by layered closure.

PCBM from the iliac crest was packed firmly in the alveolar cleft, and the mesh was then fixed using a titanium screw. The PCBM weight grafted to the cleft was regarded as the grafted bone mass. When there was excess PCBM, the difference between the surplus and harvested PCBM weights was regarded as the grafted bone mass. As for wound closure, a horizontal incision was made in the oral vestibule to reduce tension so that the labial mucoperiosteum could cover the mesh without tension, and watertight closure of the surgical site was performed (Figures 1 and 2).

\section{$\mathrm{X}$-ray and $\mathrm{CT}$ imaging}

Bisector occlusal X-ray and dental small exposure field X-ray CT (3DX Multi-Image Micro CT FPD 8; Morita Co., Tokyo, Japan; 3DX) images were obtained in the cleft area 6 months postoperatively. During 3DX, ear axes were inserted into the ears to keep the Frankfort plane horizontal. Projection data were collected by rotating the X-ray machine $360^{\circ}$ around the patients for $17.8 \mathrm{~s}$ (tube voltage $90 \mathrm{kV}$; tube current $5 \mathrm{~mA}$ ) to obtain sagittal, frontal, and axial images. The range of the present machine was a $40 \times 40 \mathrm{~mm}$ column. Slice width during CT scanning was $0.08 \mathrm{~mm}$. The image was reconstructed with an output slice width of $0.25 \mathrm{~mm}$

\section{Evaluation of bone bridge shape on X-ray and CT images}

Bone bridge shape was evaluated in 2 and 3 dimensions. For 2D evaluation, occlusal X-ray images taken 6 months postoperatively were used for evaluation, in accordance with the classification method of Enemark et al. which classifies the height of the bone bridging alveolar crest using a 4-point system (score 1: $>75 \%$; score 2: $50 \%$; score 3: $25 \%$; score $4: \leq 25 \%$ ) (Figure 3) [9].

In contrast, cleft width, thickness, and vertical height of the bone bridge were evaluated using CT images. Alveolar cleft width was evaluated using CT images taken 6 months postoperatively. The

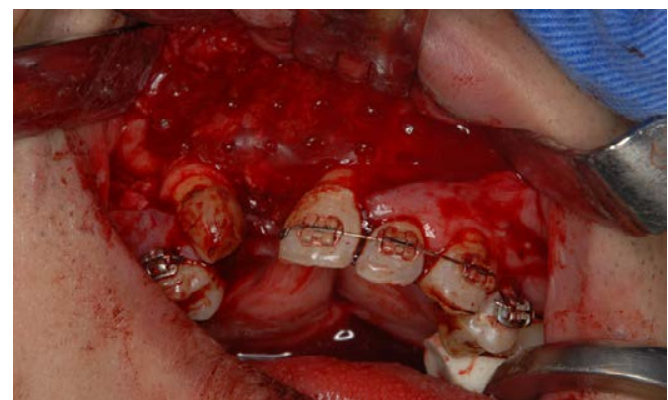

PCBM is packed into the alveolar cleft, covered with PLLA-PGA mesh, and stabilized with PLLA-PGA screws.

Figure 1: Secondary bone graft in the alveolar cleft with PLLA-PGA mesh.

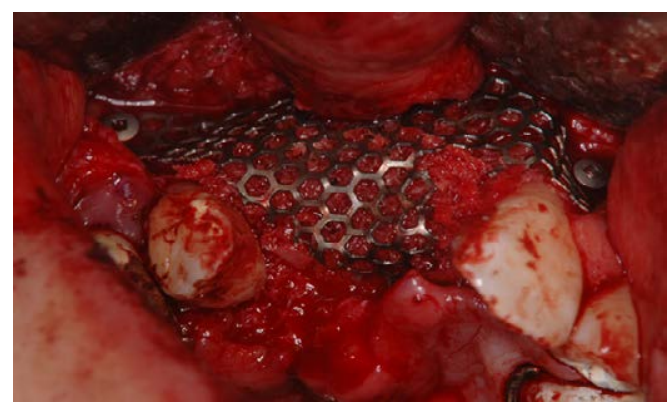

Titanium mesh is placed over the PCBM packed in the cleft and stabilized with titanium screws.

Figure 2: Secondary bone graft in the alveolar cleft with titanium mesh.

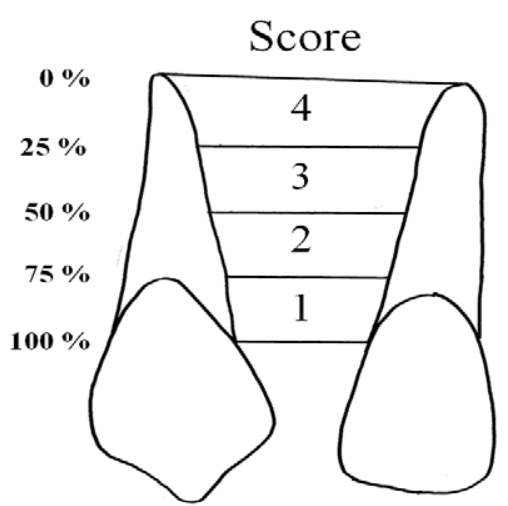

Figure 3: Score for marginal bone level assessed on intraoral films. 
difference in permeability was used to distinguish between existing and new bones. The lower margin of the nasal aperture was regarded as the upper margin of the alveolar cleft, and the alveolar crest adjacent to the alveolar cleft was regarded as the lower margin of the alveolar cleft. The width of the area located in the middle vertically where bilateral existing bones were the closest on the horizontal slicing image was measured as the alveolar cleft width (Figure 4). The anteroposterior thickness of the area located in the center of the sagittal slice located in the mesiodistal center of the alveolar cleft was measured as the thickness of the bone bridge (Figure 5). The vertically thickest area of the bone bridge was measured on the same image used for the bone bridging thickness measurement as the height of the bone bridge (Figure 6). Each measurement was performed 3 times, and the average was used as the measurement value.

\section{Statistical analysis}

The statistical software package $\mathrm{R}$ (version 2.12.0) was used. For two continuous objective variables, i.e., bone bridge thickness and height obtained from the CT image, analysis of covariance (ANCOVA) with model-simplification procedures was performed to obtain minimal adequate models [10,11]. For modeling of the scores for bony bridge alveolar crest height obtained from occlusal X-ray image analysis, a generalized linear model (GLM) was used with the binomial error structure and the logit link function, since the score

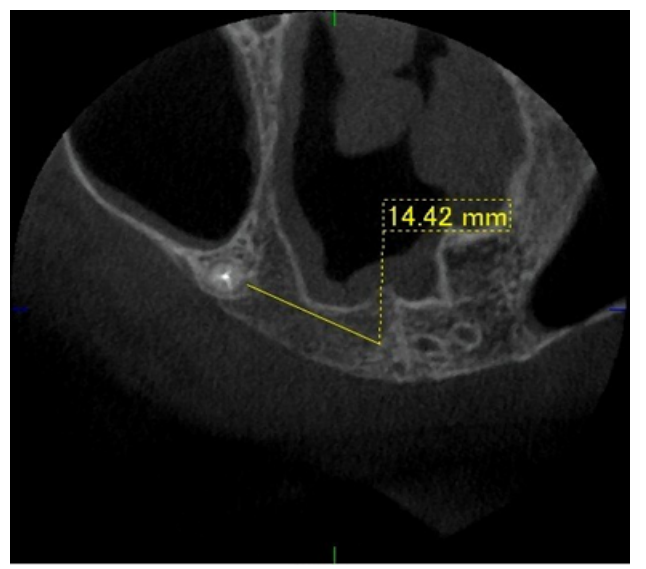

Figure 4: Measurement of alveolar cleft width evaluated on CT images.

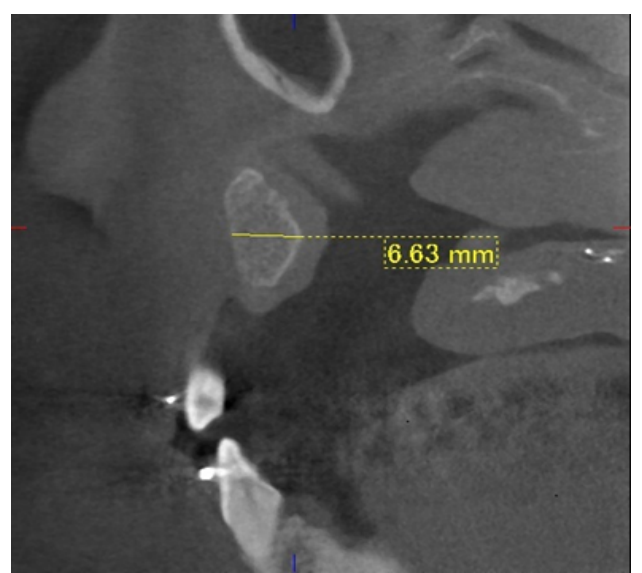

Figure 5: Measurement of bone bridge thickness.

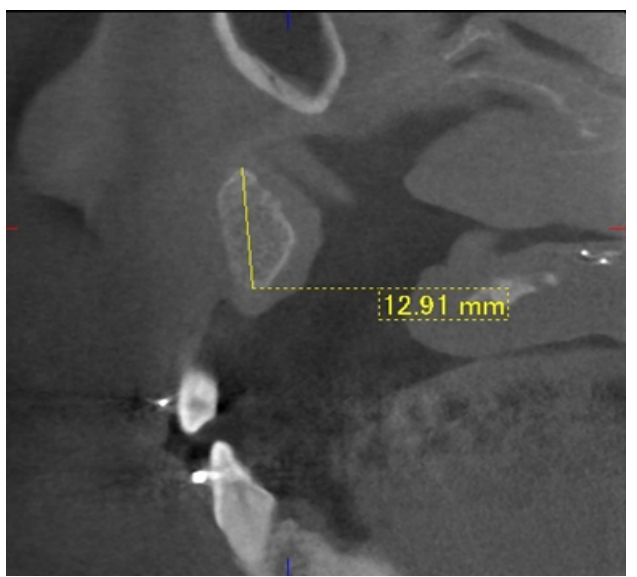

Figure 6: Measurement of bone bridge height.

took integer values strictly bound from 1 to 4 . This setting for GLM is known to be useful for analyzing data for proportions [11]. Since the scores were determined based on the proportion of the height of the bone bridging alveolar crest as described previously, this setting was selected from various combinations of error structures and link functions for GLM. In these three analyses, mesh type, sex, age, grafted bone mass, and alveolar cleft width measured on CT images were used as five explanatory variables for building first maximal models, and subsequent model simplification procedures were performed to remove non-significant variables from the model. In the simplification, the best minimal combination of explanatory variables that minimized Akaike's information criterion (AIC) was explored, and each of the remaining variables was examined for whether it was needed in the minimal adequate model with a significance level of $5 \%[10,11]$.

\section{Results}

\section{Clinical process}

There were no abnormal findings, such as divulsion of the wound, mesh exposure, or local infection, during observation.

\section{Measurement results}

PCBM weight grafted to the alveolar cleft area ranged from 2.8 to $10.0 \mathrm{~g}$ (mean $\pm \mathrm{SD}, 5.91 \pm 2.19 \mathrm{~g}$ ). The width of the cleft jaw on CT images ranged from 7.15 to $16.20 \mathrm{~mm}(12.40 \pm 2.66 \mathrm{~mm})$. The grade classification of the alveolar crest based on 6-month postoperative occlusal X-ray images included 7 cases of grade 4, 3 cases of grade 3 , and 2 cases of grade 2 . In contrast, the thickness of the bone bridge on CT images ranged from 4.55 to $13.92 \mathrm{~mm}(9.35 \pm 3.19 \mathrm{~mm})$. The height of the bone bridge on CT images ranged from 7.71 to $15.53 \mathrm{~mm}(11.42$ $\pm 2.81 \mathrm{~mm}$ ) (Table 1$)$.

\section{Results of ANCOVA and GLM analysis}

For ANCOVA with bone bridge thickness as the objective variable, the process began by fitting the maximal model with all five explanatory variables, and then the model was simplified in terms of AIC. As a result, the best model was the one using only the mesh type as a categorical explanatory variable $(\mathrm{AIC}=27.09)$. However, the test using this model revealed that mesh type was not a significant factor affecting bone bridge thickness ( $\mathrm{p}=0.086$ ) (Figure 7).

On the other hand, the results of ANCOVA with bone bridge height as the objective variable showed that a simplified model using 


\begin{tabular}{|c|c|c|c|c|c|c|c|}
\hline Type of Mesh & Sex & Age & $\begin{array}{l}\text { Grafted Bone } \\
\text { Mass (g) }\end{array}$ & $\begin{array}{c}\text { Alveolar Cleft Width } \\
(\mathrm{mm})\end{array}$ & $\begin{array}{l}\text { Marginal Bone Level } \\
\text { (Score) }\end{array}$ & $\begin{array}{l}\text { Bone Bridging Thickness } \\
\qquad(\mathrm{mm})\end{array}$ & $\begin{array}{l}\text { Bone Bridging Height } \\
(\mathrm{mm})\end{array}$ \\
\hline PLLA-PGA Mesh & $\mathrm{F}$ & 14 & 5.2 & 16.2 & 1 & 5.3 & 13.5 \\
\hline PLLA-PGA Mesh & $M$ & 22 & 4.2 & 11.95 & 2 & 9.43 & 9.97 \\
\hline PLLA-PGA Mesh & $\mathrm{F}$ & 15 & 2.8 & 8.98 & 1 & 4.55 & 15.53 \\
\hline PLLA-PGA Mesh & M & 30 & 7.4 & 14.42 & 3 & 6.63 & 12.91 \\
\hline PLLA-PGA Mesh & $\mathrm{F}$ & 30 & 4.7 & 11.15 & 2 & 7.63 & 11.44 \\
\hline PLLA-PGA Mesh & $M$ & 34 & 6.5 & 15.02 & 1 & 13.07 & 15.42 \\
\hline Titanium Mesh & $\mathrm{F}$ & 14 & 5.0 & 10.73 & 1 & 13.92 & 8.28 \\
\hline Titanium Mesh & $\mathrm{F}$ & 18 & 6.7 & 7.15 & 1 & 9.25 & 12.43 \\
\hline Titanium Mesh & $M$ & 17 & 3.7 & 13.46 & 1 & 11.44 & 7.71 \\
\hline Titanium Mesh & $\mathrm{F}$ & 35 & 5.6 & 11.43 & 3 & 12.06 & 9.25 \\
\hline Titanium Mesh & M & 54 & 8.6 & 14.42 & 1 & 6.63 & 12.91 \\
\hline Titanium Mesh & $\mathrm{F}$ & 21 & 10.6 & 13.93 & 2 & 12.25 & 7.71 \\
\hline
\end{tabular}

F: Female; M: Male

Table 1: Subjects and measurement results.

only mesh type was the best model ( $\mathrm{AIC}=21.58)$, and the test showed that mesh type was a significant factor affecting bone bridge height $(p=0.027)$. The estimated mean for each mesh type obtained from ANCOVA showed that the height was greater when using PLLA-PGA mesh than when using titanium mesh (Figure 8).

The GLM analysis of the score for bony bridge alveolar crest height showed that all five explanatory variables should be removed during the simplification procedures, and there were no significant factors affecting the score. Standard ANCOVA procedures with the score as the objective variable showed the same results as GLM analysis.

\section{Discussion}

Secondary bone grafting in the bone defect area of the alveolar cleft was reported by Boyne and Sands in the 1970s, and it has since been considered an important treatment for patients with cleft lip and palate to obtain a continuous dental arch, to guide permanent teeth in the cleft jaw area, and to form an anatomical alveolar bone shape [1]. In recent years, CT has been used to three-dimensionally evaluate the treatment results of bone bridge shape [12-14]. However, it is difficult to use CT in daily practice due to high radiation exposure levels. In the present study, 3DX was used for 3D evaluation of bone bridge shape. The radiation exposure level of 3DX is approximately the same as that of a conventional panoramic X-ray. The skin radiation exposure level of $3 \mathrm{DX}$ is $1 / 100$ of that of helical CT $(160 \mathrm{mSV})[15,16]$. Synchronized cross-sectional imaging that shows continuous sagittal, frontal, and axial planes is possible in $3 \mathrm{DX}$. This enables $3 \mathrm{D}$ visualization of the newly-formed bone bridge. It also allows the border between new and existing bone to be distinguished, and therefore, the shape of the alveolar cleft before bone grafting can be evaluated. In addition, the accuracy of length measurement on 3DX is higher than that of CT. Therefore, $3 \mathrm{DX}$ is considered useful for the $3 \mathrm{D}$ evaluation of small and complex areas such as the alveolar cleft.

The prognosis of orthodontic movement of adjacent teeth and implant treatment in the bone grafted area is affected by the vertical and labio-lingual bone mass of the bone bridge. Therefore, 3D postoperative evaluation is essential after bone grafting in the alveolar cleft. However, conventional evaluation after bone grafting using occlusal X-ray images is $2 \mathrm{D}$. In the present study, an occlusal X-ray image was used to evaluate the alveolar crest height in the bone grafting area using 4 grades according to the method of Enemark et al [9].

The usefulness of absorbable mesh with regard to the shape of the bone bridge formed in the alveolar cleft area was evaluated by comparison between titanium and absorbable meshes. Conventionally, $3 \mathrm{D}$ evaluation of the bone bridge using medical CT has been performed by measuring bone bridge volume $[13,14]$. However, the slice width of 3DX is $0.08 \mathrm{~mm}$, while that of CT is $0.625 \mathrm{~mm}$. Due to the extremely thin slice width of $3 \mathrm{DX}$, volume analysis is rough when compared with medical CT images. In addition, bone thickness and height measurable on 3DX images were evaluated, since the vertical and labio-lingual bone masses of the bone bridge are important in the evaluation of bone grafting area. The 3DX image was created by reconstructing and outputting the radiographic data.

Postoperative 3DX images were used for the measurement of alveolar cleft width, together with anatomical indices, such as adjacent teeth of the alveolar cleft and the inferior border of the nasal aperture and radiolucency, to enhance measurement reproducibility. Alveolar cleft width estimation and bone bridge measurement on 3DX images and grade classification using an occlusal X-ray require techniques such as tracing, and it is therefore impossible to completely eliminate subjective errors. To avoid errors between examiners, the same examiner was therefore in charge of analyzing the diagnostic criteria in all cases. In addition, bone grafting in the alveolar cleft was performed by the same operator. The risk of errors caused by differences in the 3DX image evaluator and doctor in charge was thus eliminated, and reproducible results were obtained.

The influences on bone bridge formation were analyzed using ANCOVA. The results showed that sex, age, grafted bone mass, alveolar cleft width, and mesh type were not significant factors affecting bone bridge thickness. ANCOVA of the factors affecting bone bridge height showed that only mesh type was a significant factor, and there was a tendency for greater bone bridging to be seen with the PLLA-PGA mesh. GLM analysis of the alveolar crest height in the alveolar cleft on occlusal X-ray images revealed that there were no significant factors. These results suggest that the shape of the bone bridge in the alveolar cleft is not affected by mesh type (PLLA-PGA mesh or titanium mesh) during bone grafting in the alveolar cleft. The PLLA-PGA mesh is considered to be more patient-friendly than the titanium mesh because it is bioabsorbable, and therefore, does not require further surgery for removal.

The number of cases was small in the present preliminary study, and a randomized, controlled trial that includes a control non-mesh group, uses age matching, and considers the cleft factors in the evaluation is necessary to clarify the effectiveness of PLLA-PGA mesh for bone 
Citation: Shirota T, Ogura H, Suzuki M, Akizuki A, Kamatani T, et al. (2016) Clinical Evaluation of Bioabsorbable Mesh for Secondary Bone Grafts in the Alveolar Cleft. Dentistry 6: 353. doi:10.4172/2161-1122.1000353

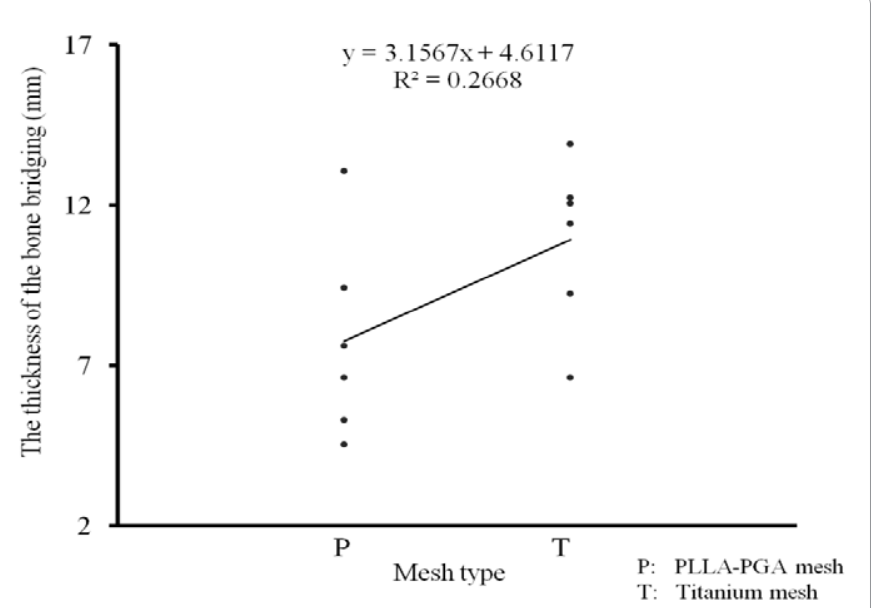

Figure 7: Mesh type is not a significant factor affecting bone bridge thickness.

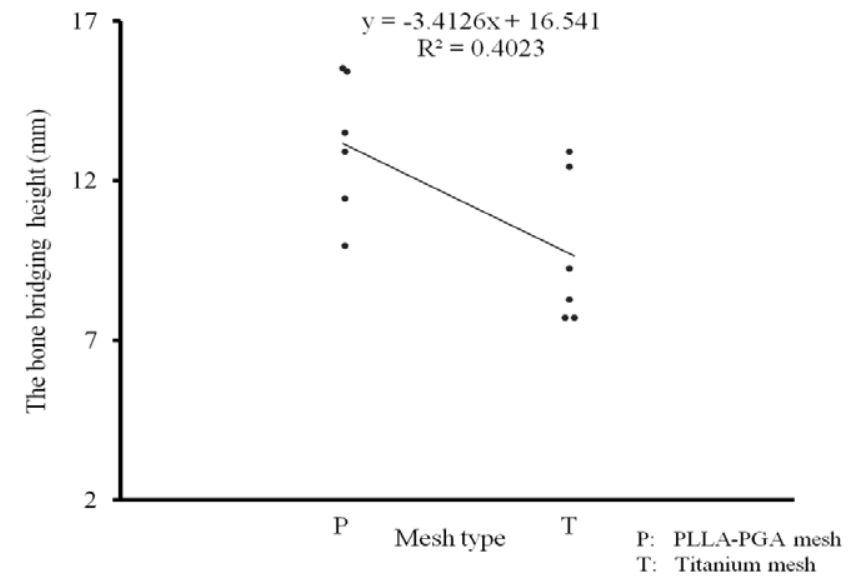

Figure 8: Bone bridge height is greater when using PLLA-PGA mesh than when using titanium mesh.

grafting in the alveolar cleft for permanent dentition cases requiring alveolar shape formation.

\section{Conclusion}

The results of the study indicate that there were no marked differences in bony bridge morphology in the alveolar cleft area between the PLLA-PGA and titanium mesh groups. The PLLA-PGA mesh is considered to be more patient-friendly than the titanium mesh because it is bioabsorbable, and therefore, does not require further surgery for removal.

\section{References}

1. Boyne PJ, Sands NR (1972) Secondary bone grafting of residual alveolar and palatal clefts. J Oral Surg 30: 87-92.

2. da Silva Filho OG, Teles SG, Ozawa TO, Filho LC (2000) Secondary bone graft and eruption of the permanent canine in patients with alveolar clefts: literature review and case report. Angle Orthod 70: 174-178.

3. Pieri F, Corinaldesi G, Fini M, Aldini NN, Giardino R, et al. (2008) Alveolar ridge augmentation with titanium mesh and a combination of autogenous bone and anorganic bovine bone: A 2-year prospective study. J Periodontol 79: 20932103.

4. Roccuzzo M, Ramieri G, Spada MC, Bianchi SD, Berrone S (2004) Vertical alveolar ridge augmentation by means of a titanium mesh and autogenous bone grafts. Clin Oral Implants Res 15: 73-81.

5. Matsui Y, Ohta M, Ohno K, Nagumo M (2006) Alveolar bone graft for patients with cleft lip/palate using bone particles and titanium mesh: A quantitative study. J Oral Maxillofac Surg 64: 1540-1545.

6. Ueki K, Okabe K, Miyazaki M, Mukozawa A, Moroi A, et al. (2011) Skeletal stability after mandibular setback surgery: comparisons among unsintered hydroxyapatite/poly-l-lactic acid plate, poly-L-lactic acid plate, and titanium plate. J Oral Maxillofac Surg 69: 1464-1468.

7. Edwards RC, Kiely KD, Eppley BL (2001) The fate of resorbable poly-L-lactic/ polyglycolic acid (LactoSorb) bone fixation devices in orthognathic surgery. J Oral Maxillofac Surg 59: 19-25.

8. Hollier LH, Rogers N, Berzin E, Stal S (2001) Resorbable mesh in the treatment of orbital floor fracture. J Craniofac Surg 12: 242-246.

9. Enemark H, Sindet-Pedersen S, Bundgaard M (1987) Long-term results after secondary bone grafting of alveolar clefts. J Oral Maxillofac Surg 45: 913-919.

10. Crawley MJ (2005) Statistics: An introduction using R. John Wiley \& Sons Ltd, Chichester, England.

11. Crawley MJ (2007) The R book. John Wiley \& Sons Ltd, Chichester, England.

12. Van der Meij AJ, Baart JA, Prahl-Andersen B, Valk J, Kostense PJ, et al. (2001) Bone volume after secondary bone grafting in unilateral and bilateral clefts determined by computed tomography scans. Oral Surg Oral Med Oral Pathol Oral Radiol Endod 92: 136-141.

13. Ozawa T, Omura S, Fukuyama E, Matsui Y, Torikai K, et al. (2007) Factor influencing secondary alveolar bone grafting in cleft lip and palate patients: prospective analysis using CT image analyzer. Cleft Palate Craniofac J 44: 286-291.

14. Freichtinger M, Zemann W, Mossböck R, Kärcher H (2008) Three-dimensiona evaluation of secondary alveolar bone grafting using a 3D-navigation system based on computed tomography: a two-year follow-up. $\mathrm{Br} \mathrm{J}$ Oral Maxillofac Surg 46: 278-282.

15. Terakado M, Hashimoto K, Arai Y, Honda M, Sekiwa T, et al. (2000) Diagnostic imaging with newly developed ortho cubic super-high resolution computed tomography (Ortho-CT). Oral Surg Oral Med Oral Pathol Oral Radiol Endod 89: 509-518.

16. Honda K, Larheim TA, Johannessen S, Arai Y, Shinoda K, et al. (2001) Ortho cubic super-high resolution computed tomography: A new radiographic technique with application to the temporomandibular joint. Oral Surg Oral Med Oral Pathol Oral Radiol Endod 91: 239-243. 